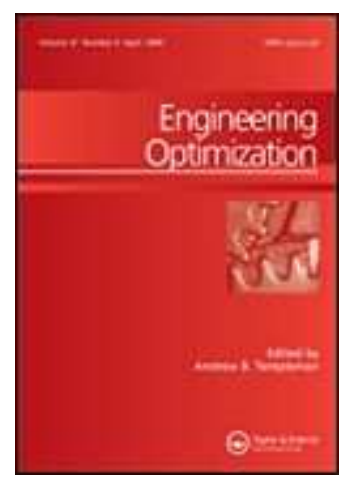

\title{
Discrete Tolerance Allocation for Product Families
}

\begin{tabular}{|r|l|}
\hline Journal: & Engineering Optimization \\
\hline Manuscript ID: & GENO-2010-0104.R3 \\
\hline Manuscript Type: & Original Article \\
\hline Date Submitted by the \\
Author: & 28 -Jan-2011 \\
\hline Complete List of Authors: & $\begin{array}{l}\text { Lööf, Johan; Chalmers University of Technology, Product and } \\
\text { production development } \\
\text { Söderberg, Rikard; Chalmers University of Technology, Product and } \\
\text { production development }\end{array}$ \\
\hline Keywords: & Tolerance allocation, Discrete optimization, Product families \\
\hline
\end{tabular}

\section{SCHOLARONE ${ }^{m}$ Manuscripts}




\title{
Discrete Tolerance Allocation for Product Families
}

\author{
Johan Lööf* \\ Rikard Söderberg
}

Product and Production Development, Chalmers University of Technology, Göteborg, Sweden

*Corresponding author. Email: johan.loof@chalmers.se

Received 12 May 2010; final version received 28 January 2011

\begin{abstract}
This article extends earlier research on the discrete tolerance allocation problem in order to optimize an entire product family simultaneously. This methodology enables a top-down tolerancing approach where requirements at assembly level on products within a family are allocated to single part requirements. The proposed solution has been implemented as an interface with an optimization algorithm coupled with a variation simulation software. The article also consists of an extensive review on the discrete tolerance allocation problem which motivates the extension of the discrete tolerance allocation problem. The suggested approach has been applied to a robot family consisting of two variants with the same requirements at product level.
\end{abstract}

Keywords: Tolerance allocation; Discrete optimization; Product Families

\section{Introduction}

Many companies today often have product families with products that are in different product segments. This is often achieved with modularity and efficient product architectures and with the use of sharing components across the organization in the different products. It is also done between different brands and companies that share common platforms. The research within the field of product families and product platforms is an extensive area, and for a thorough review see Jiao et al., (2007). Within a product family there are products sharing components, and therefore it is important to perform simulations in a module-based way. The benefits of this are to perform simulations for the whole product family simultaneously and to consider effects on the family if a common part has had a design change. When performing simulations there are many aspects that one needs to consider, but this article only covers the area of managing the effects of geometrical variation within a product family, especially focused on tolerance allocation.

Edholm and Söderberg (2009b) described a module-based way to work with variation simulation models for product families. They also described methods to manually optimize 
tolerances and locator concepts for a part or sub-assembly for all environments in a family simultaneously. Thereby the effects of geometrical variations on the assembly and on the final product can be minimized for all variants in the product family. They also described how locator information can be stored and used in a PLM (Product Lifecycle Management) environment for easier definition of simulation models (Edholm and Söderberg, 2009a). Izquierdo et al. (2009) considered fixture layout optimization for a product family with the objective to minimize the combined sensitivity of the products to fixture variation.

The scope of this research is to extend the possibilities of earlier research on tolerance allocation. Tolerance allocation implies a decomposition of an allowed variation (product tolerance) in a certain critical product dimension onto dimensions contributing to this variation. This article aims to extend the tolerance allocation methodology to be able to consider selection of tolerances in a number of products within a product family simultaneously. Since products are sharing common parts, it is important that this is considered when tolerances are defined. This is preferably done with the aid of optimization. Other benefits of this type of optimization are to choose among suppliers that are able to produce a part within a specific tolerance at a certain cost. Tseng and Huang (2009) considered a multi-plant tolerance allocation model assigning manufacturing operations at different plants in order to minimize the total manufacturing cost. This idea is extended in this article so that it is suitable for optimizing a whole product family simultaneously. Limitations in this article were that only rigid assemblies were considered and only tolerance values were allocated. In order to automatically define geometrical tolerance types the method needs to be further developed by for example incorporating different tolerance libraries. There is also a need to develop an extended method on how to calculate variation contributions from different tolerance types. 


\section{Managing geometrical variation}

Many companies today have special processes for managing geometrical variation, often defined as a geometry assurance process according to Söderberg et al. (2006). This process is divided into three phases: the concept phase, verification phase and production phase. In the concept phase, the product and the production concept are developed. Product concepts are analyzed and optimized to withstand the effect of manufacturing variation, and tested virtually against available production data. In this phase, the concept is optimized with respect to robustness and verified against assumed production systems by statistical tolerance analysis.

Product tolerances are allocated down to part and fixture level. In the verification phase and pre-production phase, the product and the production system are physically tested and verified. Adjustments are made to both product and production system to correct errors and prepare for full production. In this phase, inspection preparation takes place. This is the activity in which all inspection strategies and inspection routines are decided. In the production phase, all production process adjustments are completed and the product is in full production. Focus in this phase is on controlling production and detecting and correcting errors. The actual phase considered in this article is the concept phase where locating schemes are set and tolerances must be allocated.

\subsection{Locating Schemes}

Before any simulations can be performed on how geometrical variation propagates, one needs to know how the variation propagates in an assembly. This can be calculated in a number of different ways; see Shen et al. (2005). In this article, locating schemes are used to define how the variation propagates through an assembly. The most common locating scheme used in industry is the 3-2-1 principle. It works as follows (see Figure 1): three primary points, A1, $\mathrm{A} 2$ and A3, represent a plane that locks two rotations and one translation; two secondary 
points, B1 and B2, lock one rotation and one translation; and a tertiary point, $\mathrm{C} 1$, locks the final translation.

When defining locating schemes, it is preferable to make them as robust as possible. This means that the locators should be placed in such a way that variation in the locators does not amplify the variation in product dimensions. Söderberg (1998) presented how this could be done in a computer-aided tolerancing tool (CAT). Locator positions have also been defined using optimization to obtain a robust locating scheme. This implies that the total robustness of a part is optimized by placing the locators in an optimal way. Wang et al. presented algorithms with the objective to minimize the positional variances due to locator setup errors (Wang 1999a, b, Wang 2000, Wang and Perlinescu 2001). Lööf et al. (2009) developed a strategy to optimize the positions of locators to maximize robustness in critical product dimensions. Making a design as robust as possible can also be interpreted as making the design as uncoupled as possible (Suh, 1990).

When the robust locating schemes are defined, tolerances are allocated on the locators in the locating scheme and on other dimensions. Simulations are now conducted to investigate whether the total variation that arises from the variation in the locators and other contributing dimensions is under statistical control. This is often referred as tolerance stack-up, tolerance analysis or variation simulation.

\subsection{Tolerance Allocation}

In an early design phase, nominal values are assigned to each dimension of the product (Figure 2). According to fundamental rules for dimensioning and tolerancing, each dimension shall have a tolerance (Moy, 1964). Tolerance allocation implies an automatic assignment of tolerances to each dimension. This can be achieved by using different optimization strategies, while at the same time fulfilling the requirements on allowed variation (product tolerance) in different defined product dimensions. Upper bounds on the variation of each dimension are 
found from these requirements. The requirements can either be functionally or geometrically related.

The task is then to specify tolerances such that (a) these bounds are not violated and (b) a certain property is optimized. This property does not necessarily have to coincide with the manufacturing cost in any way; it could be a purely geometric characteristic that is desired to be minimized/maximized. Figure 2 illustrates a simple assembly consisting of three parts, $\mathrm{a}, \mathrm{b}$ and $\mathrm{c}$. The assembly has a dimension $\mathrm{D}$ that is critical and has a certain allowed variation $\mathrm{T}$ that has to be fulfilled. Tolerance allocation implies that the dimensions that contribute to the allowed variation $\mathrm{D}$ in the assembly should be assigned with a tolerance value automatically.

However, it is also preferable to choose tolerances in such a way that the manufacturing cost is minimized. A fundamental subject within this area is that tighter tolerances imply higher costs due to more complex and time-demanding manufacturing processes. When allocating tolerances it is important that the allowed variation in product dimensions is under control. In the literature there are three different ways of defining a tolerance allocation problem with the aim of minimizing the manufacturing cost.

As shown in Figure 3 they are: (1) using only continuous variables, Equation 1; (2) mixing both discrete and continuous variables, Equation 2; or (3) incorporating only discrete variables, Equation 3.

$$
\begin{aligned}
\min & \sum_{i} C_{i}\left(t_{i}\right) \\
& \sum_{i}\left(a_{i k} t_{i}\right)^{2} \leq T_{k}^{2} \quad k=1, \ldots, m \\
& l_{i} \leq t_{i} \leq u_{i},
\end{aligned}
$$

Equation 1. Continuous tolerance allocation problem (Chase and Parkinson, 1991). 
The continuous tolerance allocation problem has a cost function $C_{i}$ available for each tolerance $i$. The sensitivity coefficient $a_{i k}$ measures how much the tolerance $t_{i}$ contributes to the accumulation of variation in product dimension $k$. Tolerances are allowed to be allocated within a tolerance band $\left(l_{i}, u_{i}\right) . T_{k}$ is the allowed variation in the product dimension $k$ that must not be exceeded. Many different cost functions have been suggested which define the relationship between the cost and the size of the tolerance. A numerous selection of cost functions has appeared in the literature; see for example Chase and Parkinson (1991). If one of these functions describes the true manufacturing cost correctly, solving the optimization problem will indeed result in a minimized production cost for the manufacturer. Wu et al. (1988) made a summary of different solution approaches to this problem.

Instead of using only one cost function, several functions can be used to fit different processes when producing individual parts, defined here as the mixed discrete tolerance allocation problem. In this case, discrete variables are needed to decide which process to use when machining a certain tolerance. A continuous cost model is available for each of these machining processes. In Equation 2, $n$ is the number of dimensions that shall be assigned with a tolerance, and $p_{i}$ is the number of available processes for tolerance $i . X$ is a binary variable which ensures that only one process is chosen for each tolerance.

$$
\begin{aligned}
& \min \sum_{i=1}^{n} \sum_{j=1}^{p_{i}} X_{i j} C_{i j}\left(t_{i j}\right) \\
& \sum_{i=1}^{n} \sum_{j=1}^{p_{i}}\left(a_{i k} t_{i j}\right)^{2} X_{i j} \leq T_{k}^{2} \quad k=1, \ldots, m \\
& l_{i j} \leq t_{i j} \leq u_{i j} \\
& \sum_{j=1}^{p_{i}} X_{i j}=1 \quad i=1, \ldots, n \\
& X_{i j} \in\{0,1\} \quad i=1, \ldots, n, j=1, \ldots, p_{i} .
\end{aligned}
$$

Equation 2. Mixed discrete tolerance allocation problem (Chase et al., 1990). 
Chase et al. (1990) solved this optimization problem with different processes early on. The problem can also be formulated in a way allowing using multiple processes for one tolerance. Other early solutions to the problem considering multiple processes were suggested by Zhang and Wang (1993) and Gadallah and ElMaraghy (1994). A selection of other solutions involving genetic algorithms for solving the two above-discussed problems includes those of Iannuzzi and Sandgren (1994), Prabhaharan et al. (2004) and Singh et al. (2005).

Instead of using continuous functions, pure discrete tolerance cost alternatives can be used, which represent the true solution and are the focus in this research. This is motivated since it is hard to realize a proposal from a continuous solution. The discrete case is intended to be based on real data that are possible to define. The discrete tolerance allocation problem is described as follows.

$$
\begin{aligned}
& \min \sum_{i=1}^{n} \sum_{j=1}^{p_{i}} x_{i j} c_{i j} \\
& \sum_{i=1}^{n} \sum_{j=1}^{p_{i}}\left(a_{i k} t_{i j}\right)^{2} x_{i j} \leq T_{k}^{2} \quad k=1, \ldots, m \\
& \sum_{j=1}^{p_{i}} x_{i j}=1 \quad i=1, \ldots, n \\
& x_{i j} \in\{0,1\} \quad i=1, \ldots, n, j=1, \ldots, p_{i} .
\end{aligned}
$$

Equation 3: Discrete tolerance allocation problem (Ostwald and Huang, 1977).

For each dimension $i$, one should denote the available tolerance choices $t_{i j}$ together with associated costs $c_{i j}$. The index $j$ goes from 1 to the number of available alternatives $\left(p_{i}\right)$ for each tolerance $t_{i}$, and $i$ goes from 1 to the number of dimensions ( $n$ ) which shall be assigned with a tolerance value. The binary variable $x_{i j}$ ensures that only one tolerance for each dimension is assigned. Furthermore, $a_{i k}$ is the sensitivity coefficient for tolerance $t_{j}$ with respect to the product dimension number $k$. The objective function (3a) minimizes the total cost. The budget constraint (3b) ensures that the limit on the variation of each of the product dimensions is not violated. (3c) is called an assignment constraint. It guarantees that exactly 
one tolerance is assigned to each dimension. (3d) ensures that $x_{i j}$ is a binary variable. The rest of this section presents a comprehensive review of earlier research on the discrete tolerance allocation problem.

An early approach to solving this problem was considered by Moy (1964), where he used dynamic programming to assign tolerances. Programming in this case is equivalent to optimization. Smathers and Ostwald (1972) divided the problem of N discrete component tolerances into $\mathrm{N}$ subprograms and then solved this by following Bellman's principle of optimality. Wilde (1975) suggested a simplification of the problem defined in Smathers and Ostwald (1972) by pseudo-Boolean programming. Ostwald and Huang (1977) formulated the problem as in Equation 3, but with the worst-case constraint instead of RSS. They used Balas's 0-1 algorithm to solve the problem. Monte and Datseris (1982) developed a software package aimed for helping designers in optimum tolerance selection. They chose a discrete combinatorial optimization technique for the optimization. Kim and Knott (1988) suggested that the methods proposed by Wilde (1975) and Ostwald and Huang (1977) could be combined to provide an improved approach. The suggested pseudo-Boolean approach allowed simplifications through a number of questions that could be asked by designers and manufacturing engineers. Wu et al. (1988) used exhaustive search to evaluate all possible combinations. At this time they pointed out that no existing zero-one programming method could guarantee an optimal solution. Lee and Woo (1989) developed a branch-and-bound algorithm for ensuring optimal selection. They insisted on real-time usage of this approach. Chase et al. (1990) compared a number of discrete optimization schemes with exhaustive search. CPU time and number of combinations required to find the global optimum were used to compare the different methods.

Kusiak and Feng (1995) compared integer programming, design of experiments and Taguchi method for the discrete tolerance allocation problem. Feng and Kusiak (1997) were 
early on incorporating the quality loss function in the discrete optimization problem. They used a stochastic integer programming to solve the discrete optimization problem. Teeravaraprug (2002) also considered loss functions and used a commercial software (LINDO) for integer programming. Feng et al. (2001) included supplier selection in the problem and used the stochastic integer programming approach to solve it. Xue and Ji (2004) developed a genetic algorithm to solve the discrete tolerance allocation problem and applied it in a two-dimensional angular tolerance charting problem.

Lööf et al. (2005) and Lööf et al. (2007) applied the Land-Doig-Dakins algorithm to the discrete optimization problem with general loss functions. For more information regarding discrete optimization and [0-1] programming please refer to Nemhauser and Wolsey (1999). Teeravaraprug (2007) compared the results of using a worst-case or an RSS constraint in the optimization problem. Lööf et al. (2008) used the discrete tolerance allocation methodology to secure variation along a robot path. Tseng and Huang (2009) considered a multi-plant tolerance allocation model with the goal to assign manufacturing operations at different plants in order to minimize the total manufacturing cost.

\section{Using discrete tolerance allocation for product families}

The research on the discrete tolerance allocation problem has only been applied to single products. The aim of this section is to illustrate how this can be applicable to product families. Products in a product family are sharing components, but this implies that - depending on where and how the components are located - they can affect the variation in critical product dimensions differently. Often when designing one product, individual tolerances can be adjusted to control the overall variation, but in the product family case one has to consider many products simultaneously. Figure 4 illustrates a simple example of two products sharing common components and where product 2 has an extra unique part. 
Product 1 and product 2 can have different or common requirements on allowed variation in certain critical dimensions on the product level. Since products within a family have common parts, it is interesting to consider this when breaking down a requirement of an allowed variation in a critical dimension on the product level onto dimensions on part level. Due to the extra unique part, the propagation of geometrical variation will be different in the two products due to the longer tolerance chain in product 2 . The propagation is often defined by using variation simulation software to define how parts are located relative to each other. When locating schemes are defined, sensitivity coefficients can be derived which define how much each locating point contributes to the total variation in critical dimensions on the product level. Since it is the locating points that control the propagation of variation, these points need to have a tolerance such that an allowed variation on the product level is under control. The optimization problem formulated in Equation 3 can be used for product families.

The information can be stored in a similar way but with extended information in the matrix $a$, with sensitivity coefficients (see Figure 5). The vector $V$ is extended with information about allowed variation for every critical dimension on products in the family. In the product family optimization, the indices in Equation 3 are described as follows. $n$ is the number of total dimensions that shall be assigned with a tolerance. In this case where two products are to be optimized, $n=c+u_{p 1}+u_{p 2}$, which implies the sum of common dimensions, unique dimensions for product 1 and unique dimensions for product 2 . The constant, $m=d_{p 1}+d_{p 2}$, is the total sum of critical dimensions for product 1 and product 2 . The matrix presented in (Figure 5) illustrates a family consisting of two products. The matrix can easily be extended to store information about a general number of products by adding extra column and rows. 


\subsection{Case study}

The method described above has been applied to a robot family consisting of two variants illustrated in (Figure 6). In this case a requirement on an allowed variation in the tool centre point within the $\mathrm{x}, \mathrm{y}, \mathrm{z}$ directions is considered, which implies a total of six critical dimensions, three for each robot. The differences of the robots are illustrated in (Figure 6), where the right robot has an extra part which extends the length of the robot and also makes the tolerance chain longer. CAD models of the robots have been imported into a variation simulation software where locating schemes have been defined between the parts to define the accumulation of variation. In this case only tolerances in the interfaces between the robot parts are considered. This implies tolerances on the locating points in locating schemes defining how the parts are connected and how variation accumulates. Another thing that has to be clarified is that each A-plane consists of 3 points, and each point has different sensitivity coefficients. Since these three points lay in the same plane and often in the same physical plane, one can reduce these three points to one tolerance with one sensitivity coefficient for each discrete step and direction. So the three sensitivity coefficients for the A plane are reduced to one by using root sum squares. The same procedure yields for the two B-points. The locating scheme concepts between the robot components are illustrated in Figure 7, this concept holds for all the interfaces.

Totally the robots have fifteen common tolerances and nine unique tolerances, of which robot 1 has three and robot 2 has six unique tolerances. The next step is to use optimization to assign each tolerance with a value. The optimization algorithm used to solve the problem is presented in Lööf et al. (2007). The method has also been applied to securing an allowed variation along a specified robot path; see Lööf et al. (2008).

The input to the dialog window is the 6 critical dimensions and 24 tolerances with sensitivity coefficients for each of the 6 critical dimensions. The critical dimensions and the tolerance that shall be defined with a value are specified in the CAT software, and the sensitivity 
coefficients are calculated numerically through a high-mean-low analysis by the variation simulation software RD\&T (Robust Design and Tolerancing) (RD\&T, 2010). When this is done, a requirement on an allowed variation for each critical dimension is defined. In this case the requirement was $\pm 0.5 \mathrm{~mm}$ for all the six dimensions. Finally, a number of alternative values and costs for each tolerance are to be defined. This is done inside the optimization dialog window. In this case eight alternative values have been defined for each tolerance. These alternatives are presented in Table 2, and represent both internal and external manufacturing possibilities. One can also define a unique number of available values for each tolerance that shall be assigned with a value. Once all data are available, the optimization can be performed. The result from the optimization presented in Table 3 shows that all critical dimensions are within the range of allowed variation and at the same time the cost is optimized. If the two variants are optimized separately, the output will deviate from the family optimization. Due to the shorter tolerance chain in robot 1 , the optimization yields wider tolerance values and the result is closer to the range of $1 \mathrm{~mm}$. Applying results from this optimization to robot 2 will result in exceeding the allowed limit of variation. Optimizing robot 2 can correlate with the result from family optimization due to the similarities of robot 1 and robot 2. This is not always the case when common parts can be located in different environments with different types of accumulation in the assemblies.

\section{Conclusion}

From the literature study it is concluded that discrete tolerance allocation has not been applied to product families earlier. The general formulation has been extended to be able to optimize the selection of tolerances fulfilling overall requirements on different products sharing parts in a product family. In the product family case, this implies that tolerances on common parts must be chosen to fit all environments where the part is used. The authors motivate their choice of the discrete definition with the fact that it is possible to represent the true cost of 


\section{References}

Chase, K. W., Loosli, B. G., Greenwood, W. H. \& Hauglund, L. F. (1990) Least Cost Tolerance Allocation for Mechanical Assemblies with Automated Process Selection. Manufacturing Review, Vol 3, No. 1., pp. 49-59.

Chase, K. W. \& Parkinson, A. R. (1991) A Survey of Research in the Application of Tolerance Analysis to the Design of Mechanical Assemblies. Research in Engineering Design, 3, 23-27.

Edholm, P. \& Söderberg, R. (2009a) Locator Management for Platform-Based Architectures in PLM Systems. Proceedings of the 11th International Conference on Computer Aided Tolerancing, March 26-27, Annecy, France.

Edholm, P. \& Söderberg, R. (2009b) Module-based variation simulation in platform architecture. Proceedings of the 11th International Conference on Computer Aided Tolerancing, March 26-27, Annecy, France.

Feng, C.-X. \& Kusiak, A. (1997) Robust Tolerance Design With the Integer Programming Approach. Journal of Manufacturing Science and Engineering, November 1997, Vol. 119 ,

Feng, C.-X., Wang, J. \& Wang, J.-S. (2001) An Optimization Model for Concurrent Selection of Tolerances and Suppliers. Computers \& Industrial Engineering, 40 (2001), 15-33.

Gadallah, M. H. \& Elmaraghy, H. A. (1994) A New Algorithm for Discrete Tolerance Optimization. Proceedings of the Fourth International Conference on Computer Integrated Manufacturing and Automation Technology. Troy, NY, USA, 10-12 Oct 1994.

Iannuzzi, M. \& Sandgren, E. (1994) Optimal Tolerancing: The Link Between Design and Manufacturing Productivity. Design Theory and Methodology; ASME DTM.

Izquierdo, L. E., Hu, S. J., Du, H., Jin, R., Jee, H. \& Shi, J. (2009) Robust Fixture Layout Design for a Product Family Assembled in a Multistage Reconfigurable Line. Journal of Manufacturing Science and Engineering, 131, 
Jiao, J. R., Simpson, T. W. \& Siddique, Z. (2007) Product family design and platform-based product development: a state-of-the-art review. Journal of Intelligent Manufacturing, 18, 5-29.

Kim, H. S. \& Knott, K. (1988) A Pseudo-Boolean Approach to Determining Least Cost Tolerances. International Journal of Production Research, Vol 26. No.1., pp. 157167.

Kusiak, A. \& Feng, C.-X. (1995) Deterministic Tolerance Synthesis: A Comparative Study. Computer Aided Design, Vol 27, No. 10., October 1995, 759-768.

Lee, W.-J. \& Woo, T. C. (1989) Optimum Selection of Discrete Tolerances. Journal of Mechanisms, Transmission, and Automation in Design, ASME 111, 243-251.

Lööf, J., Hermansson, T. \& Söderberg, R. (2005) An Efficient Solution To The Discrete-Least Cost Tolerance Allocation Problem With General Loss Functions. In proceedings of The 9th International Seminar on Computer Aided Tolerancing. 10-12 April, Tempe, Arizona, USA.

Lööf, J., Hermansson, T. \& Söderberg, R. (2007) An Efficient Solution To The Discrete-Least Cost Tolerance Allocation Problem With General Loss Functions. IN DAVIDSON, J. K. (Ed.) Models for Computer Aided Tolerancing in Design and Manufacturing. pp. 115-124, Springer.

Lööf, J., Söderberg, R. \& Lindkvist, L. (2008) Secure Variation along a Robot Path using Discrete Tolerance Allocation Optimization. Proceedings of the 2nd CIRP Conference on Assembly Technologies and Systems, 21-23 September 2008, Toronto, Ontario, Canada.

Lööf, J., Söderberg, R. \& Lindkvist, L. (2009) Optimizing Locator Position to Maximize Robustness in Critical Product Dimensions. Proceedings of the ASME 2009 International Design Engineering Technical Conferences \& Computers and Information in Engineering Conference IDETC/CIE 2009. 30 August - 2 September 2009, San Diego, California, USA.

Monte, M. E. \& Datseris, P. (1982) Optimum Tolerance Selection for Minimum Manufacturing Cost and Other Criteria. ASME Article No. 82-DET-35, 1-9.

Moy, W. A. (1964) Assignment of Tolerances by Dynamic Programming. Machine Design, 21 May, 1964, 215-218.

Nemhauser, G. L. \& Wolsey, L. A. (1999) Integer and combinatorial optimization, John Wiley \& Sons.

Ostwald, P. \& Huang, J. (1977) A Method for Optimal Tolerance Selection. Journal of Engineering for Industry, ASME, 99, 558-565.

Prabhaharan, G., Asokan, P., Ramesh, P. \& Rajendran, S. (2004) Genetic-Algorithm-Based Optimal Tolerance Allocation Using a Least-Cost Model. The International Journal of Advanced Manufacturing Technology, 24, 647-660.

Rd\&T (2010), http://www.rdttech.se/, 2010-09-14.

Shen, Z., Ameta, G., Shah, J. J. \& Davidson, J. K. (2005) A Comparative Study Of Tolerance Analysis Methods. Journal of Computing and Information Science in Engineering, 5, 247-256.

Singh, P. K., Jain, S. C. \& Jain, P. K. (2005) Comparative Study of Genetic Algorithm and Simulated Annealing for Optimal Tolerance Design Formulated with Discrete and Continuous Variables. Journal of Engineering Manufacture, 219, 735-758.

Smathers, E. W. \& Ostwald, P. (1972) Optimization of Component Functional Dimensions and Tolerances. ASME Article No. 72-DE-18.

Suh, N. P. (1990) The Principles of Design., New York, Oxford University Press.

Söderberg, R. (1998) Robust Design by Support of CAT Tools. ASME Design Engineering Technical Conference. 13-16 September, Atlanta, GA. 
Figure 1. 3-2-1 locating scheme.

Figure 2. Tolerance allocation.

Figure 3. Cost-tolerance data.

Figure 4. Product family example.

Figure 5. Matrix "a" with sensitivity coefficients.

Figure 6. Robot family.

Figure 7. 3-2-1 locating scheme applied to robot interfaces.

Figure 8. Optimization dialog window. 
Table 1. Available tolerances that shall be assigned with values.

Table 2. Tolerance alternatives.

Table 3. Accumulated variation from optimized tolerances. 

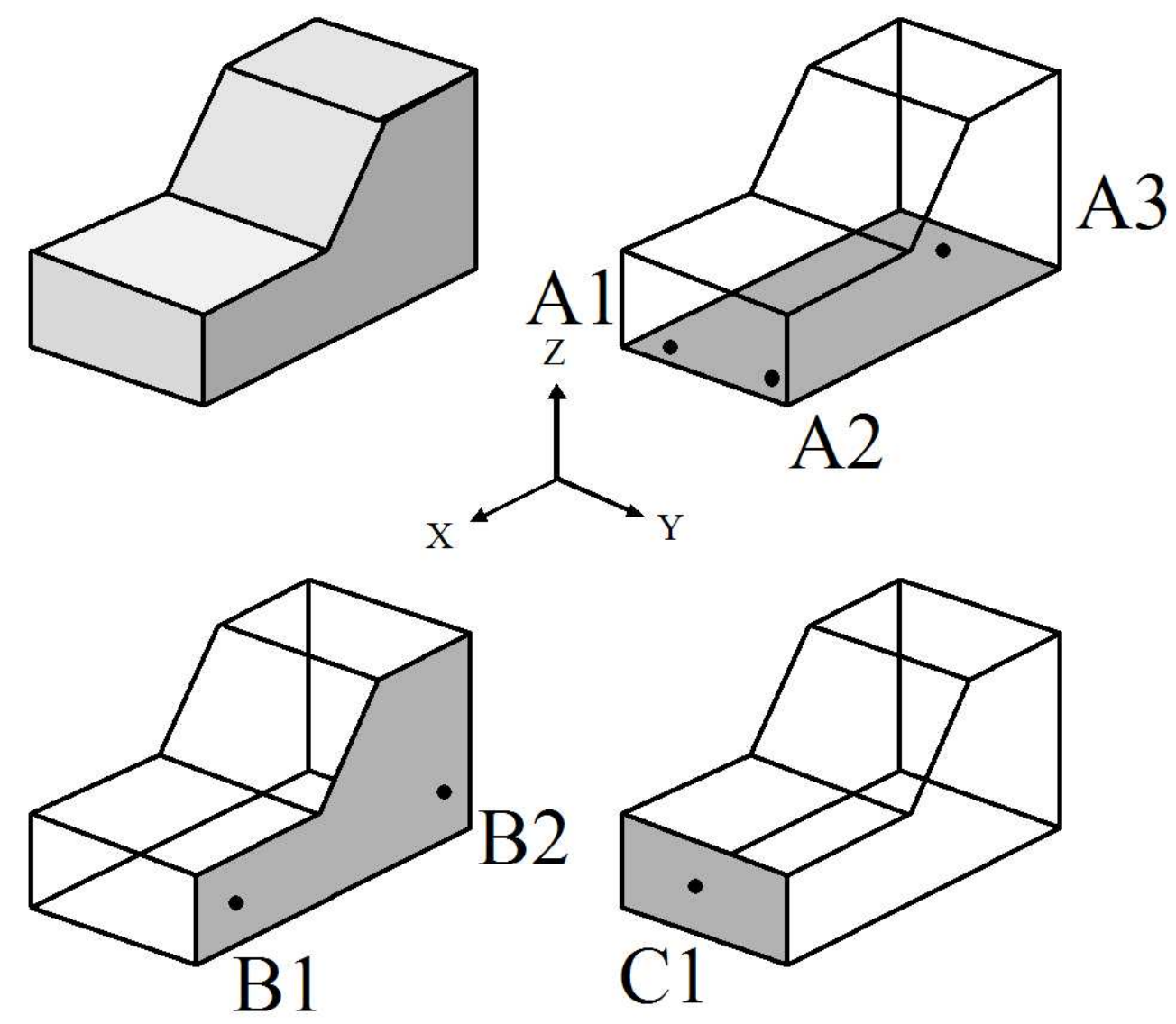

Figure 1. 3-2-1 locating scheme. $260 \times 237 \mathrm{~mm}$ (96 x 96 DPI) 
Figure 2. Tolerance allocation. $264 \times 87 \mathrm{~mm}$ (96 x 96 DPI) 


1
2
3
4
5
6
7
8
9
10
11
12
13
14
15
16
17
18
19
20
21
22
23
24
25
26
27
28
29
30
31
32
33
34
35
36
37
38
39
40
41
42
43
40
45
49
50
50
51
53
55
50

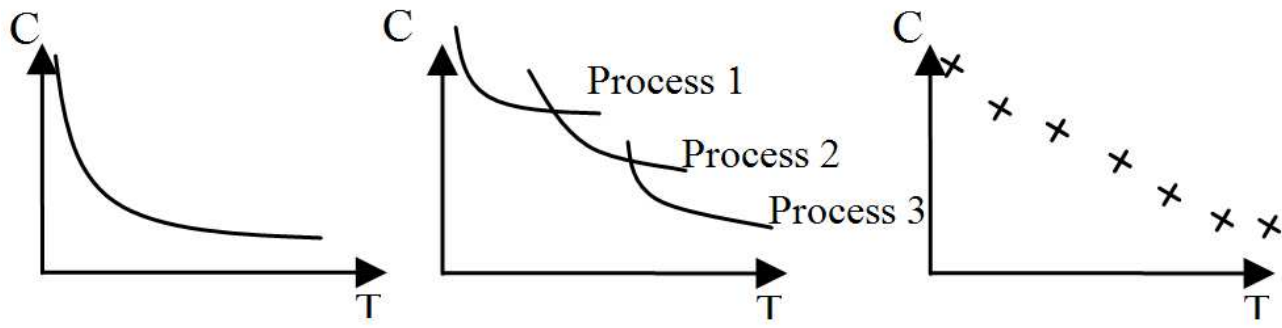

Figure 3. Cost-tolerance data. $261 \times 70 \mathrm{~mm}$ (96 × 96 DPI) 


\section{Product Family}
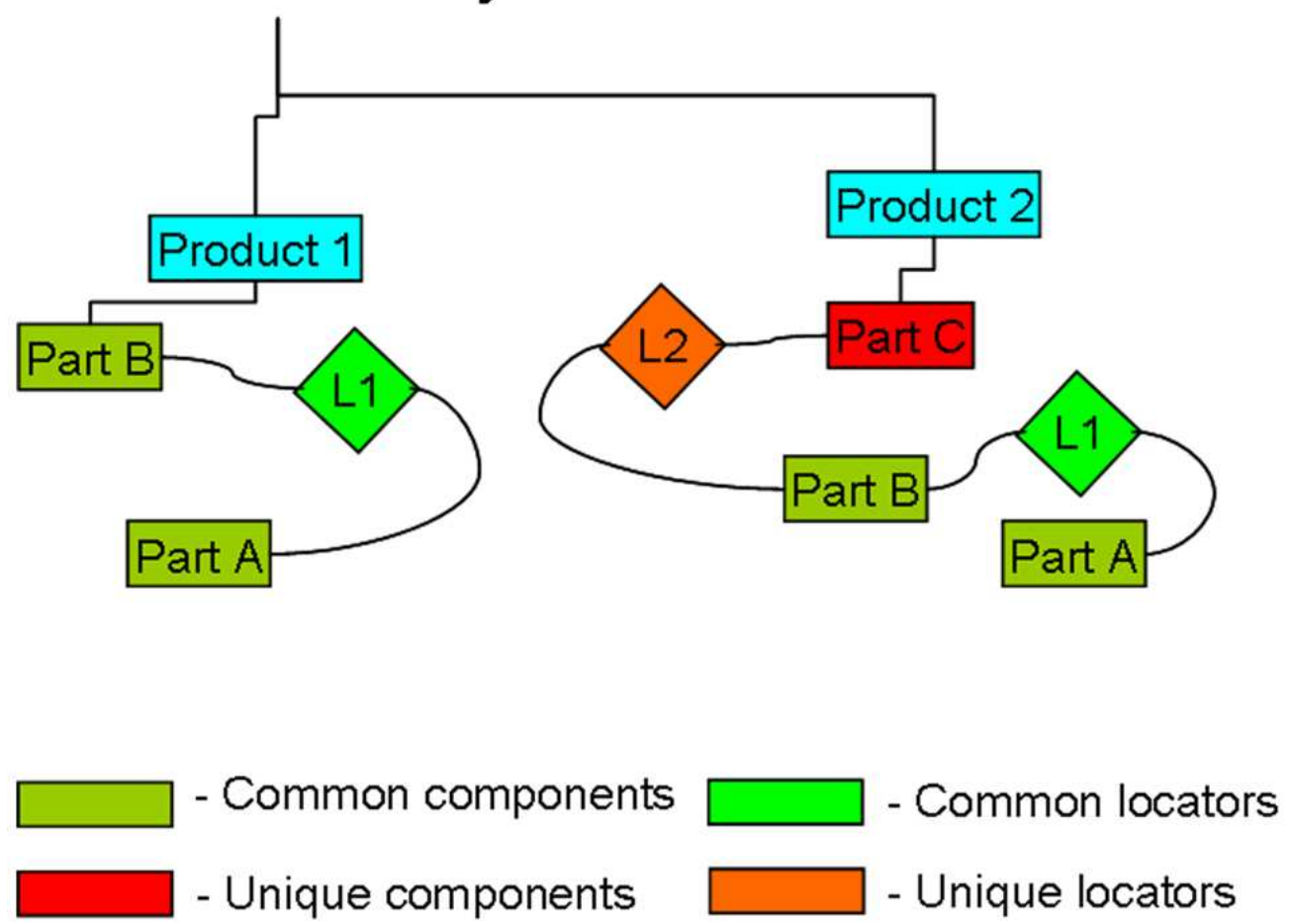

Figure 4. Product family example. $256 \times 203 \mathrm{~mm}(96 \times 96$ DPI) 


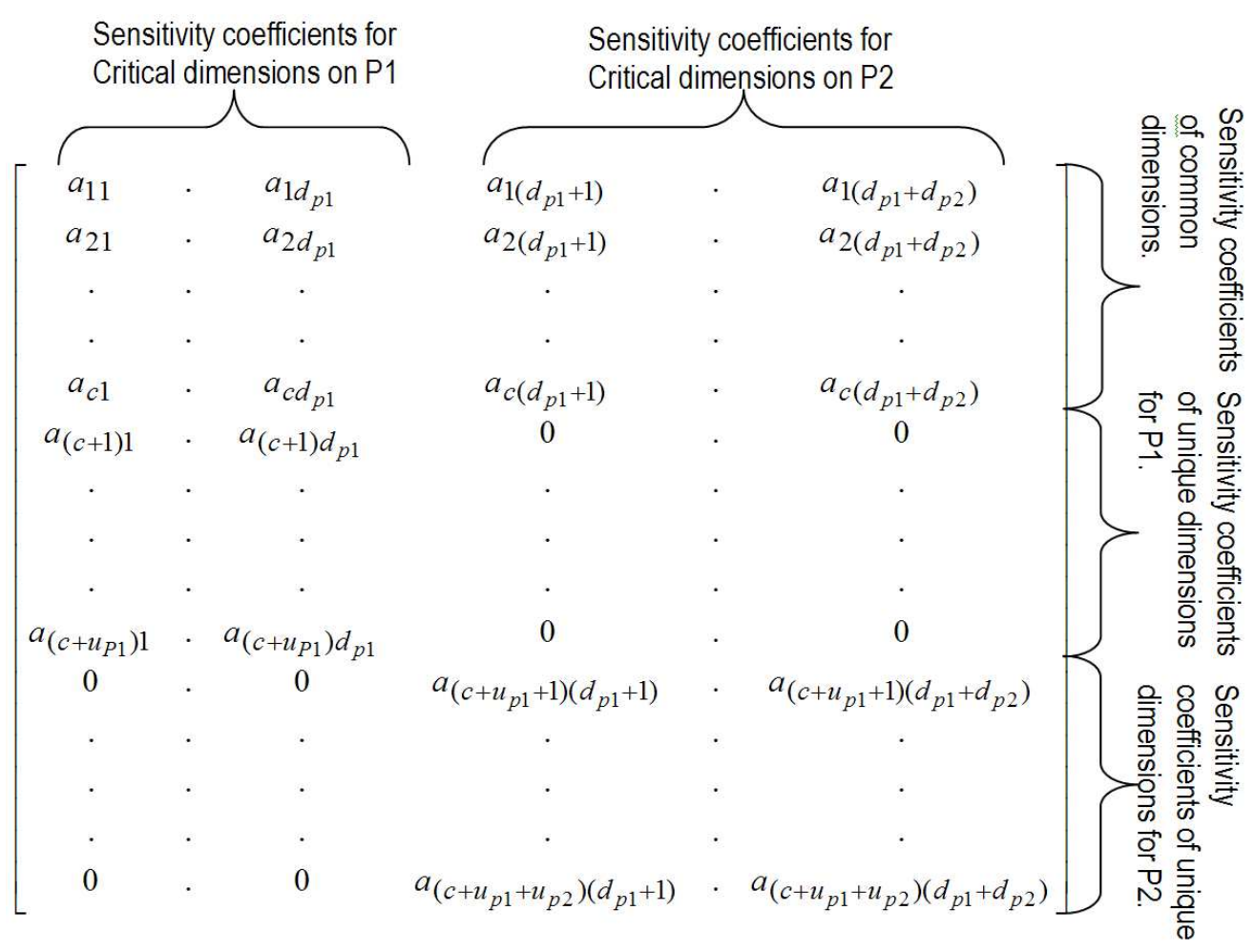

Figure 5. Matrix "a" with sensitivity coefficients.

$318 \times 241 \mathrm{~mm}(96 \times 96 \mathrm{DPI})$ 
Figure 6. Robot family. $258 \times 138 \mathrm{~mm}(120 \times 120 \mathrm{DPI})$ 


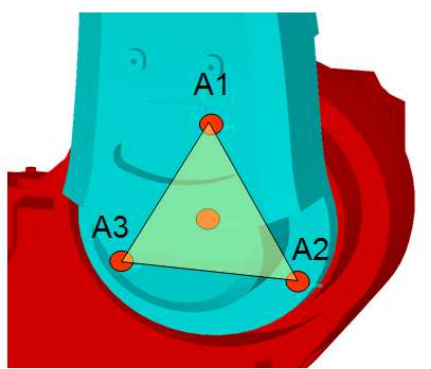

$A 1, A 2$ and $A 3$ defines the plane.

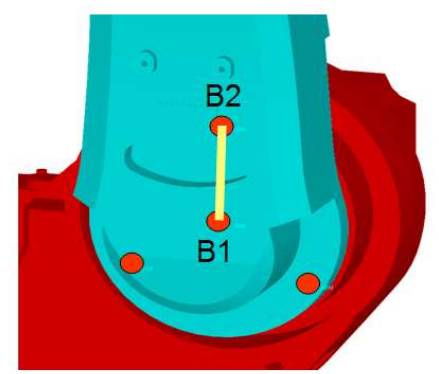

B1 and B2 defines the line.

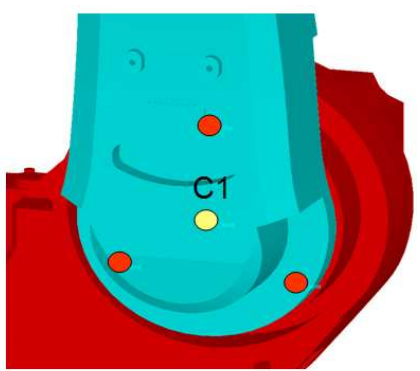

C1 defines the point.

Figure 7. 3-2-1 locating scheme applied to robot interfaces. $260 \times 90 \mathrm{~mm}(120 \times 120$ DPI $)$ 


\begin{tabular}{|l|l|l|l|l|l|}
\hline \multicolumn{2}{|l|}{ Tolerances Robot 1 } & \multicolumn{2}{l|}{ Tolerances Robot 2 } \\
\hline Base_Stand_A & Base_Stand_B & Base_Stand_C & Base_Stand_A & Base_Stand_B & Base_Stand_C \\
\hline Stand_Arm_A & Stand_Arm_B & Stand_Arm_C & Stand_Arm_A & Stand_Arm_B & Stand_Arm_C \\
\hline Arm_House_A & Arm_House_B & Arm_House_C & Arm_House_A & Arm_House_B & Arm_House_C \\
\hline House_Axle_A & House_Axle_B & House_Axle_C & House_Dist_A & House_Dist_B & House_Dist_C \\
\hline Axle_Tilt_A & Axle_Tilt_B & Axle_Tilt_C & Dist_Axle_A & Dist_Axle_B & Dist_Axle_C \\
\hline Tilt_Plate_A & Tilt_Plate_B & Tilt_Plate_C & Axle_Tilt_A & Axle_Tilt_B & Axle_Tilt_C \\
\hline & & & Tilt_Plate_A & Tilt_Plate_B & Tilt_Plate_C \\
\hline
\end{tabular}

Table 1. Available tolerances that shall be assigned with values.

$309 \times 97 \mathrm{~mm}(96 \times 96 \mathrm{DPI})$ 


\begin{tabular}{|l|c|c|c|c|c|c|c|c|}
\hline Tolerance & 0.01 & 0.02 & 0.03 & 0.04 & 0.06 & 0.08 & 0.1 & 0.2 \\
\hline Cost & 5 & 4 & 3 & 2.5 & 2 & 1.75 & 1.5 & 1.25 \\
\hline
\end{tabular}

Table 2. Tolerance alternatives. $193 \times 26 \mathrm{~mm}(96 \times 96 \mathrm{DPI})$ 


\begin{tabular}{|c|c|c|c|c|c|c|}
\hline & R1X & R1Y & R1Z & R2X & R2Y & R2Z \\
\hline Value & 0.508 & 0.940 & 0.932 & 0.507 & 0.991 & 0.999 \\
\hline
\end{tabular}

Table 3. Accumulated variation from optimized tolerances. $167 \times 27 \mathrm{~mm}(96 \times 96 \mathrm{DPI})$ 